\title{
Una formulación matemática y de solución para programar cirugías con restricciones de recursos humanos en el hospital público
}

\author{
A mathematical formulation and solution to schedule surgeries \\ with human resource constraints in a public hospital \\ Lorena Pradenas Rojas $^{1} \quad$ Exequiel Matamala Vergara ${ }^{1}$ \\ Recibido 24 de mayo de 2010, aceptado 13 de julio de 2012 \\ Received: May 24, 2010 Accepted: July 13, 2012
}

\begin{abstract}
RESUMEN
Actualmente, los hospitales públicos nacionales e internacionales presentan demandas que sobrepasan la capacidad de atención, lo que ha provocado un creciente interés por usar herramientas de gestión en los centros clínicos que les permita realizar de forma eficiente y eficaz la entrega de servicios a los distintos pacientes. El presente estudio aporta una nueva forma de abordar el problema de programación de cirugías, desde la programación matemática, presentando un modelo de optimización multiobjetivo y un algoritmo metaheurístico implementado computacionalmente, que permite la programación semanal de intervenciones quirúrgicas, cumpliendo con los requerimientos de pabellones y personal especializado necesario para su realización. Se utiliza una instancia de prueba, donde el tiempo de ejecución del algoritmo, implementado en $\mathrm{C}++$, fue de siete minutos para 191 cirugías en lista de espera. El tiempo alcanzado es considerablemente menor a la programación realizada con un sistema manual, como los actualmente usados en hospitales públicos.
\end{abstract}

Palabras clave: Programación de cirugías, programación multiobjetivos, asignación de personal, algoritmos genéticos, gestión de salud.

\section{ABSTRACT}

Currently, national and international public hospitals have demands that exceed their service capacity, which has caused a growing interest in management sciences to deliver these medical centers the tools that will enable them to perform efficiently and effectively, delivering services to different patients. This study provides a new way of approaching the problem of surgical scheduling using mathematical programming, presenting a multi-objective optimization model and a metaheuristic algorithm implemented computationall. The solution allows weekly schedule of surgical procedure and complying with the requirements of the flag and expertise necessary for realization. We ordered test instances where the execution time of the algorithm, coded in C++, was 7 minutes for a 191 surgeries waiting list, which is a considerable less amount of time to this schedule than using a manual approach. The latest is the case of public hospitals.

Keywords: Surgeries scheduling, multiobjective programming, staffing, genetic algorithms, health management.

\section{INTRODUCCIÓN}

El problema de programar cirugías en hospitales públicos nacionales continúa sin disponer de soluciones eficientes que permitan utilizar de la mejor forma posible los recursos de salud que disponen y que además proporcione un buen servicio a los usuarios. En particular, que permita a cada paciente tener su intervención lo más pronto posible y en las mejores condiciones, para evitar un agravamiento

1 Departamento de Ingeniería Industrial. Universidad de Concepción. Concepción, Chile.E-mail: lpradena@udec.cl; ematamala@udec.cl 
de su enfermedad, y disponer una calidad de vida apropiada.

En el actual sistema de salud público en Chile, cuando una intervención quirúrgica no se realiza en el tiempo considerado por el Ministerio de Salud, se debe trasladar a centros privados o cancelar las multas estipuladas. En ambos casos el costo involucrado es extraordinariamente elevado y perjudica el buen uso de los recursos disponibles.

A diferencia de los países desarrollados, en Chile aún no se dispone de herramientas de planificación eficientes que permitan realizar una adecuada gestión de los recursos disponibles en las instituciones de salud. La gestión de recursos es de gran importancia, ya que algunos pacientes pueden fallecer antes de ser intervenidos $y$, en otros, los tiempos de espera superan los seis meses.

El uso de herramientas y algoritmos computacionales apoyarían la gestión de estas instituciones y como consecuencia proporcionarían un mejor uso de los recursos y una mejor atención a los pacientes, con todos los beneficios que esto conlleva.

El presente estudio determina si es posible modelar matemáticamente y propone una solución factible al problema de programación de cirugías en un hospital público considerando las restricciones de recursos humanos involucradas.

Problemas similares han sido tratados en la literatura especializada, entre los cuales se pueden mencionar:

- En [10] se plantea un modelo estocástico para la planificación de pabellones quirúrgicos para dos tipos de pacientes: electivos y de emergencia. Se propone un método que combina la simulación Montecarlo con Programación entera mixta. Este método garantiza soluciones factibles en tiempos computacionales razonables.

- En [13] se propone un modelo matemático de optimización multiobjetivo que permite al tomador de decisiones planificar la programación de cirugías en el plazo de un año para reducir las listas de espera. El algoritmo de solución propuesto entrega dos soluciones, una solución máxima eficiente o una solución equilibrada. Las soluciones permiten determinar si las metas fijadas al comienzo del periodo pueden ser cumplidas o no con los recursos disponibles.

- El estudio [14] tiene por objetivo establecer un marco teórico que permita al personal médico establecer la programación, según sus preferencias. Además generar una programación de las cirugías considerando las restricciones presentes en el centro médico. Se plantea un modelo matemático e implementa un algoritmo genético en un centro de salud de Bélgica.

- En [2] se proporciona una amplia revisión bibliográfica y estado del arte como también se menciona un estudio de caso para la programación multiobjetivo de un día con métodos de Branch and Cut.

- En [4] se modela la programación de cirugías como un taller de pedidos ( $\mathrm{J} o b \mathrm{Job}$ ).

- En los estudios [1] y [17], nosotros hemos propuesto modelos matemáticos de programación lineal monoobjetivo. En el primer caso, maximizando el nivel de importancia asignado a cada cirugía y minimizando la diferencia entre el tiempo disponible de las salas quirúrgicas y el tiempo de utilización de las mismas, no se considera el personal necesario para la cirugía, sólo se usa la disponibilidad horaria de los pabellones. Se programan cirugías dentro de un día. En el segundo, maximizamos la suma de los niveles de urgencia de las distintas cirugías, con restricciones de los pabellones. No considera al personal especializado. La asignación de los cirujanos se realiza con un algoritmo basado en backtracking cronológico.

En las siguientes secciones del presente artículo se presentan primero los principales aspectos de los algoritmos genéticos y la optimización multiobjetivo relacionados con el tema. Posteriormente se entrega el modelo matemático propuesto, luego las características de la instancia de prueba y, finalmente, los principales resultados y conclusiones del estudio.

\section{ALGORITMOS GENÉTICOS}

Las metaheurísticas están diseñadas para abordar problemas complejos de optimización, en donde otros métodos fallan en términos de eficacia y eficiencia. Son reconocidas como enfoques muy útiles para resolver problemas reales (complejos). 
Los enfoques metaheurísticos se caracterizan por presentar estrategias de solución general, las cuales pueden ser adaptadas a diferentes situaciones.

Algunas metaheurísticas [7-16], como por ejemplo: Tabu search, genetic algorithms, simulated annealing $y$ ant colony optimization, tienen como principal desafío adaptar un problema particular al formato de éstas normalmente, requieren menos trabajo que el desarrollo de una heurística especializada.

En [8] se proponen los algoritmos genéticos; basado en el proceso de evolución y herencia que presentan los seres vivos, al reproducirse aquellos individuos que poseen mejores características, sus hijos tendrán mayores posibilidades de sobrevivir.

Como se resume en [11], un algoritmo genético dispone de cinco componentes básicos:

- Una representación genética de la solución.

- Una forma de crear una población inicial de soluciones factibles.

- Una función de evaluación (fitness) para medir la calidad de las soluciones.

- Operadores genéticos que modifican la composición genética de las soluciones, tales como: cruzamiento, mutación, selección, etc.

- Valores de los parámetros que utilizan los algoritmos genéticos (ejemplo: tamaño de la población, criterio de detención, probabilidades de la aplicación de los operadores genéticos, etc.).

Las ventajas principales del uso de algoritmos genéticos son: Adaptabilidad, se puede trabajar con cualquier tipo de función objetivo y cualquier tipo de restricciones (lineal, no lineal, discretas, etc.); Robustez, por la reducción en el esfuerzo computacional para encontrar una solución y también porque las nuevas modificaciones propuestas permiten una mayor exploración del espacio solución, evitando caer en soluciones óptimas locales.

Los operadores genéticos comúnmente utilizados son:

- Selección, basándolo en el proceso de selección natural, este operador selecciona individuos de una población, dando una mayor probabilidad de sobrevivencia a los individuos mejores en una determinada característica.
- Cruzamiento, es el principal operador genético y consiste en obtener nuevos hijos (soluciones) mediante el cruce de dos individuos pertenecientes a la población y la combinación de sus cromosomas.

- Mutación, consiste en un cambio aleatorio en los genes de un individuo, generando un nuevo individuo.

\section{PROBLEMAS DE OPTIMIZACIÓN MULTIOBJETIVO}

Los problemas de optimización multiobjetivo son problemas que presentan dos o más funciones objetivo. A diferencia con los problemas monoobjetivos, que pueden alcanzar la solución óptima, los multiobjetivos no tienen solución óptima, debido que no existe una solución que sea la mejor respecto a todos los objetivos e incluso pueden existir conflictos entre los objetivos. Una solución puede ser mejor en un objetivo, pero peor en otros. Existe un conjunto de soluciones que no se pueden comparar entre sí, este tipo de soluciones son denominadas soluciones no dominadas o soluciones de la frontera de Pareto [12].

La decisión final de cuál solución seleccionar de la frontera de Pareto depende de la perspectiva de cada tomador de decisiones. En rigor, depende de la curva de utilidad, si existe o si es posible de disponer.

Para obtener la frontera de Pareto se requiere de un tiempo computacional elevado, por esta razón se proponen técnicas metaheurísticas, las cuales han sido adaptadas para problemas multiobjetivos, son de gran apoyo en la búsqueda de buenas aproximaciones a la frontera de Pareto, una de estas técnicas son los algoritmos evolutivos.

\section{ENFOQUES DE SOLUCIÓN A PROBLEMAS MULTIOBJETIVOS}

Algunas estrategias para problemas multiobjetivos con algoritmos genéticos son:

- Vector evaluated genetic algorithm (veGA). Es uno de los primeros trabajos que utilizan algoritmos genéticos para resolver problemas de optimización multiobjetivo y propuesto en $[3,15]$. Usan distintos operadores genéticos en individuos de diferentes subpoblaciones, identificando a los individuos que entregan 
soluciones no dominadas. Se protege a los mejores individuos en uno de los objetivos y se proporcionan probabilidades adecuadas para la selección de individuos que son mejores que la media en más de un objetivo. Una de las dificultades que presenta es la convergencia a subregiones de la frontera de Pareto (speciation), por lo cual normalmente veGA no entrega soluciones satisfactorias.

- Multiobjective genetic algorithm (moGA). Propuesto en [5], establece una priorización (rank) a un individuo presente en la población. A todos los individuos no dominados se les asigna rank igual a 1, mientras que a los individuos dominados se les sanciona, dependiendo del número de individuos en la población actual que los dominan.

- Random-weight genetic algorithm (rwGA). En [9] se propone una suma ponderada para la evaluación de los objetivos (fitness) y obtener variabilidad en la búsqueda de la frontera de Pareto. Se asignan pesos a cada función objetivo y combinando en una única función objetivo. Los pesos se asignan al azar para evitar speciation (agrupamiento de las soluciones en una sola área de la frontera de Pareto).

- Adaptive-weight genetic algorithm (awGA): En [6] se propone un enfoque de adaptación de pesos, usando información de la población actual para reajustar los pesos, en la búsqueda de la frontera de Pareto.

\section{MODELO MATEMÁTICO}

El modelo matemático propuesto en este estudio considera definiciones y supuestos que son mencionados a continuación.

\section{CARACTERÍSTICAS DE LOS PABELLONES QUIRÚRGICOS}

La unidad de pabellones proporciona horas pabellón a los distintos servicios clínicos y en los distintos días hábiles de una semana.

Los pabellones forman grupos en los cuales los distintos servicios clínicos pueden realizar sus intervenciones; estos grupos están disponibles en días previamente establecidos por los servicios clínicos en conjunto con la unidad de apoyo a pabellones quirúrgicos. Un servicio clínico no puede utilizar horas de pabellón asignado a otro servicio.
Algunos servicios clínicos disponen de salas de uso exclusivo y pueden ser utilizadas durante todo el periodo disponible en la semana para realizar intervenciones quirúrgicas.

Las salas quirúrgicas poseen el equipamiento necesario para realizar las cirugías dependiendo de cada especialidad.

\section{TIPOS DE PACIENTES}

Se consideró a las cirugías tipo electivas, las que permiten establecer una lista de espera, con tiempos máximos, que no pueden ser sobrepasados ya que la salud del paciente se deteriora en la medida que avanza el tiempo y también las instituciones son multadas al no cumplir los tiempos establecidos. Las cirugías del tipo emergencias deben ser realizadas inmediatamente y no son consideradas en el modelo ya que se dispone de pabellones exclusivos las 24 horas y los 365 días del año.

\section{CARACTERÍSTICAS DE LAS CIRUGÍAS}

Las cirugías presentan las siguientes características:

- Una vez iniciada, la cirugía no puede ser interrumpida, no existe el concepto de paciente en proceso o con grado de avance, por lo cual las variables de decisión a considerar son enteras.

- Las cirugías requieren de pabellones que cumplan con sus necesidades particulares de equipamiento, por lo cual no todos los pabellones pueden ser utilizados para la realización de una determinada cirugía.

- Cada intervención quirúrgica debe ser realizada por cirujanos especialistas, los cuales son asignados por cada servicio clínico. Se debe disponer de personal de apoyo, tales como: enfermeras, anestesistas, auxiliar de anestesia, pabelloneras, arsenaleras y en algunos casos uno o dos cirujanos asistentes.

\section{CARACTERÍSTICAS DEL PERSONAL}

- Los cirujanos presentes en una cirugía no pueden ser asignados de forma paralela a otras cirugías, esto es similar para los auxiliares de anestesia y arsenaleras.

- Las enfermeras y anestesistas, por escasez de personal, pueden ser asignados de forma paralela a lo más a dos cirugías, pero existen cirugías 
que necesitan de enfermeras y anestesistas fijos (o exclusivos).

El modelo matemático propuesto en este estudio es un modelo de optimización multiobjetivo, considerando tres objetivos: Maximizar la sumatoria de los valores de urgencia para las distintas cirugías, maximizar la sumatoria de los tiempos de espera acumulados por las cirugías candidatas y maximizar la suma del número de cirugías realizadas.

Los objetivos anteriores presentan generalmente conflictos ya que al buscar (por ejemplo) sólo maximizar las urgencias se puede obviar a las cirugías con menor urgencia pero con un mayor tiempo de espera de los pacientes respecto de las anteriores.

Considerando lo mencionado previamente se propone el siguiente modelo matemático:

\section{FUNCIONES OBJETIVO}

$$
\begin{gathered}
M A X \quad Z_{1}=\sum_{i} \sum_{p} \sum_{d} U R G_{i} * C_{i p}^{d} \\
M A X \mathrm{Z}_{2}=\sum_{i} \sum_{p} \sum_{d} T E_{i} * C_{i p}^{d} \\
M A X \quad Z_{3}=\sum_{i} \sum_{p} \sum_{d} C_{i p}^{d}
\end{gathered}
$$

\section{RESTRICCIONES}

$$
\sum_{d} \sum_{p} C_{i p}^{d} \leq 1 \quad \forall i
$$

$$
\begin{array}{cc}
\sum_{j} C I_{j i}^{t l}=\sum_{p} C_{i p}^{d} * R C I_{i}^{t} & \forall i, d, t \\
\sum_{a} \sum_{p} A_{\text {aip }}^{d}=\sum_{p} C_{i p}^{d} * R A_{i} & \forall i, d
\end{array}
$$$$
\sum_{l} A A_{l i}^{d}=\sum_{p} C_{i p}^{d} * R A A_{i} \quad \forall i, d
$$$$
\sum_{e} \sum_{p} E_{e i p}^{d}=\sum_{p} C_{i p}^{d} * R E_{i} \quad \forall i, d
$$$$
\sum_{r} A R_{r i}^{d}=\sum_{r} \sum_{p} C_{i p}^{d} * R A R_{r}, \forall i, d
$$$$
\sum_{b} P A_{b i}^{d}=\sum_{b} \sum_{p} C_{i p}^{d} * R P A_{b}, \forall i, d
$$

$$
\begin{aligned}
& \sum_{i \in \eta} \sum_{p} C_{i p}^{d} \leq C A M_{d}^{k} \quad \forall d, k \\
& \sum_{d} C_{i p}^{d}=0 \quad i \in \xi \wedge p \in \psi \\
& \sum_{p} C_{i p}^{d}=0 \quad d \in \phi \quad \wedge \quad i \in \zeta \\
& \sum_{i} T C_{i} * C_{i p}^{d} \leq H P_{p}^{d} \quad \forall d, p \\
& \sum_{i} T C_{i} * C I_{j i}^{t l} \leq H C I_{j}^{t d} \quad \forall d, j, t \\
& \sum_{i} T C_{i^{\prime}} * E_{e i^{\prime} p}^{d}+\sum_{i^{\prime \prime}} 2 T C_{i^{\prime \prime}} * E_{e i^{\prime \prime} p}^{d} \leq 2 H E_{e}^{d} \\
& \forall d, e \quad i^{\prime} \in \varpi i^{\prime \prime} \in \omega \quad(\varpi \cap \omega=\varnothing \wedge \varpi \cup \omega=i) \\
& \sum_{i} T C_{i^{\prime}} * A_{a i^{\prime} p}^{d}+\sum_{i^{\prime \prime}} 2 T C_{i^{\prime \prime}} * A_{a i^{\prime \prime} p}^{d} \leq 2 H A_{a}^{d} \\
& \forall d, a \quad i^{\prime} \in \varpi i^{\prime \prime} \in \omega \quad(\varpi \cap \omega=\varnothing \wedge \varpi \cup \omega=i) \\
& \sum_{p} E_{\text {eip }}^{d} \leq 1 \quad \forall d, i, e \\
& \sum_{p} A_{\text {aip }}^{d} \leq 1 \quad \forall d, i, a \\
& \sum_{i} E_{e i\left(p^{\prime}\right)}^{d} \leq \sum_{i} C_{i\left(p^{\prime}\right)}^{d} \quad \forall d, e \quad p^{\prime} \in \alpha \\
& \sum_{i} A_{a i\left(p^{\prime}\right)}^{d} \leq \sum_{i} C_{i\left(p^{\prime}\right)}^{d} \quad \forall d, a \quad p^{\prime} \in \alpha \\
& C_{i p}^{d}, C I_{j i}^{t l}, A_{\text {aip }}^{d}, A A_{l i}^{d}, E_{\text {eip }}^{d}, A R_{r i}^{d}, P A_{b i}^{d} \\
& \in\{0,1\} \forall i, p, d, t, a, l, e, r, b
\end{aligned}
$$

\section{VARIABLES DE DECISIÓN}

$C_{i p}^{d}= \begin{cases}1 & \text { Si cirugía } i \text { es asignada a pabellón } p \text { en día } d \\ 0 & \text { En cualquier otro caso }\end{cases}$ $C I_{j i}^{t d}=\left\{\begin{array}{c}1 \text { Si cirujano } j \text { de especialidad } t \\ \text { es asignado a cirugía } i \text { en el día } d \\ 0 \text { En cualquier otro caso }\end{array}\right.$ $A_{\text {aip }}^{d}=\left\{\begin{array}{l}1 \text { Si anestesiólogo } a \text { es asignado } \\ \text { a cirugía } i \text { en pabellón } p \text { el día } d \\ 0 \text { En cualquier otro caso }\end{array}\right.$ 


$$
\begin{aligned}
& A A_{l i}^{d}=\left\{\begin{array}{l}
1 \text { Si auxiliar de anestesia } l \text { es asignado } \\
\text { a cirugía } i \text { en el día } d \\
0 \quad \text { En cualquier otro caso }
\end{array}\right. \\
& E_{\text {eip }}^{d}=\left\{\begin{array}{l}
1 \text { Si enfermera } e \text { es asignada a cirugía } i \\
\text { en el pabellón } p \text { en el día } d \\
0 \quad \text { En cualquier otro caso }
\end{array}\right. \\
& A R_{r i}^{d}=\left\{\begin{array}{l}
1 \text { Si arsenalero } r \text { es asignado } \\
\text { a cirugía } i \text { en el día } d \\
0 \quad \text { En cualquier otro caso }
\end{array}\right. \\
& P A_{b i}^{d}=\left\{\begin{array}{l}
1 \quad \text { Si pabellonera } b \text { es asignada } \\
\text { a cirugía } i \text { en el día } d \\
0 \quad \text { En cualquier otro caso }
\end{array}\right.
\end{aligned}
$$

\section{PARÁMETROS}

$U R G_{i}$ : Nivel de urgencia de cirugía $i$.

$T E_{i}$ : Tiempo de espera acumulado de cirugía $i$.

$R C I_{i}^{t}$ : Requerimiento de cirujanos especialistas tipo $\boldsymbol{t}$ para cirugía $\boldsymbol{i}$.

$C A M_{d}^{k}:$ Número de camas tipo $\boldsymbol{k}$ disponibles en un día $\boldsymbol{d}$.

$T C_{i}$ : Duración en horas de una cirugía $\boldsymbol{i}$ (estimada) $H P_{p}^{d}$ : Horas pabellón disponibles en día $\boldsymbol{d}$ para pabellón $\boldsymbol{p}$.

$H C I_{j}^{t d}$ : Horas disponibles cirujano $j$ de especialidad $\boldsymbol{t}$ en día $\boldsymbol{d}$.

$H E_{e}^{d}$ : Horas enfermera disponibles de enfermera $\boldsymbol{e}$ en día $\boldsymbol{d}$.

$R E_{i}$ : Requerimiento de enfermeras para la cirugía $i$. $R A_{i}:$ Requerimiento de anestesiólogos para la cirugía $i$. $R A A_{i}$ : Requerimiento de auxiliares de anestesiólogo para la cirugía $i$.

$R A R_{r}:$ Requerimiento de arsenalero tipo $\boldsymbol{r}$.

$R P A_{b}$ : Requerimiento de pabellonera tipo $\boldsymbol{b}$.

\section{DESCRIPCIÓN DEL MODELO MATEMÁTICO}

(1) Maximiza la suma de los factores de urgencia de las cirugías, para priorizar a los pacientes que requieren una pronta atención.

(2) Maximiza la suma del tiempo de espera de una cirugía, priorizando a los pacientes que llevan un mayor tiempo de espera.
(3) Maximiza el número de cirugías en el periodo de programación.

(4) Una cirugía sólo debe ser asignada una única vez.

(5) Este conjunto de restricciones indican que el número de personal especializado (enfermeras, anestesistas, etc.) debe cumplir con el requerimiento de personal especialista.

(6) El número de cirugías programadas debe ser igual o menor al número de camas disponibles.

(7) Las cirugías pertenecientes a un determinado grupo $\xi$ (pacientes de un servicio clínico particular) no pueden ser programadas en pabellones p pertenecientes a un grupo $\psi$ (salas no adecuadas para el grupo $\xi$ ). Esta restricción evita que cirugías sean programadas en pabellones que no son adecuados.

(8) En los días pertenecientes a un grupo $\phi$ no pueden ser programadas las cirugías pertenecientes a un grupo $\zeta$, para evitar que cirugías provenientes de un servicio clínico sean programadas en días sin disponibilidad de pabellones quirúrgicos.

(9) El tiempo total estimado de las cirugías programadas en un día y en un pabellón específico no pueden superar la disponibilidad de pabellones.

(10)El tiempo total estimado de las cirugías programadas en un día no puede superar las horas de cirujanos disponibles en ese día.

(11) y (12) Dos veces el tiempo disponible de una enfermera $o$ anestesista debe ser mayor o igual al tiempo de cirugías del grupo $\varpi$ (no requieren de la presencia permanente de este personal) y dos veces el tiempo de cirugías del grupo $\omega$ (requieren a una enfermera y a anestesista presente durante toda la cirugía), los grupos $\varpi$ y $\omega$ son una partición del conjunto que contiene todas las cirugías. Esta restricción es necesaria debido a que enfermeras y anestesistas pueden ser programados de forma paralela en algunas cirugías.

(13) y (14) Evitan que una enfermera o anestesista sea asignado dos veces a una cirugía.

(15) y (16) Permiten que los anestesistas y enfermeras pueden ser asignados en pabellones cercanos (grupo de pabellones $\alpha$ ), permitiendo así la asignación paralela.

(17)Las variables de decisión binarias toman valores de uno o cero. 
Las soluciones propuestas para la población inicial deben cumplir con las restricciones del modelo para que sean consideradas como soluciones factibles en la población. Los objetivos presentados serán utilizados para determinar las funciones de evaluación necesarias para el operador genético de selección.

\section{INSTANCIA DE PRUEBA}

La instancia de prueba propuesta en el estudio supone nueve pabellones quirúrgicos, seis grupos de cirugías con diferentes requerimientos de pabellón y de personal. Para asignar la espera (en días) y el tiempo de las cirugías (en horas), con el fin de diferenciar en características a los distintos grupos existentes, en [13] se proponen distribuciones triangulares para determinar parámetros a través de valores históricos, no disponemos de estos valores, entonces usamos distribuciones normales. El listado total de cirugías usado en este estudio es de 191, las cuales están detalladas en la Tabla 1.

Para determinar las urgencias se utilizó una distribución uniforme U[0,1], asignando valores del 1 al 10, siendo 10 el valor de mayor urgencia, de igual forma para todos los grupos.

Los parámetros de tiempos de cirugías son establecidos a través de distribuciones normales, debido a la aleatoriedad que presenta este parámetro en particular. No es apropiado el uso de promedios como en Vidal [17].

Todos los grupos de la Tabla 1 requieren de una persona por especialidad.

Las restricciones de personal disponible en la semana son mostradas en la Tabla 2.

Las restricciones de tiempo en minutos de los pabellones en los días están en la Tabla 3.

El método propuesto tiene tres etapas: Generación de una población factible inicial, programación de las cirugías y asignación del personal especializado. Cada grupo es tratado por separado en las etapas de población y programación. En la etapa final los grupos son tratados conjuntamente.
Tabla 1. Instancia de prueba.

\begin{tabular}{|c|c|c|c|c|c|}
\hline Gr. & Pab. & Días & Cirugías & $\begin{array}{c}\text { Espera } \\
\text { (días) }\end{array}$ & $\begin{array}{l}\text { Tiempo } \\
\text { cirugía } \\
\text { (horas) }\end{array}$ \\
\hline \multirow{3}{*}{1} & 1 & Lunes & \multirow{3}{*}{28} & \multirow{3}{*}{$\mathrm{N}(25,7)$} & \multirow{3}{*}{$\mathrm{N}(3,1)$} \\
\hline & 2 & Jueves & & & \\
\hline & 3 & Viernes & & & \\
\hline \multirow{2}{*}{2} & 2 & Martes & \multirow{2}{*}{50} & \multirow{2}{*}{$\mathrm{N}(30,9)$} & \multirow{2}{*}{$\mathrm{N}(2,0.5)$} \\
\hline & 3 & Miércoles & & & \\
\hline \multirow{3}{*}{3} & 4 & Lunes & \multirow{3}{*}{30} & \multirow{3}{*}{$\mathrm{N}(20,8)$} & \multirow{3}{*}{$\mathrm{N}(4.5,1)$} \\
\hline & 5 & Miércoles & & & \\
\hline & 6 & & & & \\
\hline \multirow{3}{*}{4} & 4 & Martes & \multirow{3}{*}{25} & \multirow{3}{*}{$\mathrm{N}(25,5)$} & \multirow{3}{*}{$\mathrm{N}(3.5,1)$} \\
\hline & 5 & Jueves & & & \\
\hline & 6 & Viernes & & & \\
\hline \multirow{3}{*}{5} & 7 & Martes & \multirow{3}{*}{30} & \multirow{3}{*}{$\mathrm{N}(27,10)$} & \multirow{3}{*}{$\mathrm{N}(2.7,1)$} \\
\hline & 8 & Viernes & & & \\
\hline & 9 & & & & \\
\hline \multirow{3}{*}{6} & 7 & Lunes & \multirow{3}{*}{28} & \multirow{3}{*}{$\mathrm{N}(35,7)$} & \multirow{3}{*}{$\mathrm{N}(4.6,1)$} \\
\hline & 8 & Miércoles & & & \\
\hline & 9 & Jueves & & & \\
\hline
\end{tabular}

Tabla 2. Restricciones de personal.

\begin{tabular}{|l|c|}
\hline \multicolumn{1}{|c|}{ Tipo } & Num. \\
\hline Anestesistas & 10 \\
\hline Auxiliar anestesia & 21 \\
\hline Enfermeras & 20 \\
\hline Pabelloneras & 19 \\
\hline Arsenaleras & 19 \\
\hline Cirujano A (Grupo 1) & 8 \\
\hline Cirujano B (Grupo 2) & 10 \\
\hline Cirujano C (Grupo 3) & 9 \\
\hline Cirujano D (Grupo 4) & 9 \\
\hline Cirujano E (Grupo 5) & 8 \\
\hline Cirujano F (Grupo 6) & 10 \\
\hline Cirujano asistente A (Grupo 1) & 9 \\
\hline Cirujano asistente B (Grupo 2) & 12 \\
\hline Cirujano asistente C (Grupo 3) & 10 \\
\hline Cirujano asistente D (Grupo 4) & 12 \\
\hline Cirujano asistente E (Grupo 5) & 10 \\
\hline Cirujano asistente F (Grupo 6) & 12 \\
\hline
\end{tabular}


Tabla 3. Restricciones de pabellón.

\begin{tabular}{|c|c|c|c|c|c|}
\hline Pab. & Lunes & Martes & Miércoles & Jueves & Viernes \\
\hline 1 & 600 & 0 & 0 & 600 & 600 \\
\hline 2 & 600 & 540 & 560 & 600 & 600 \\
\hline 3 & 600 & 570 & 500 & 600 & 600 \\
\hline 4 & 590 & 610 & 610 & 650 & 590 \\
\hline 5 & 600 & 580 & 580 & 670 & 640 \\
\hline 6 & 520 & 655 & 530 & 600 & 630 \\
\hline 7 & 660 & 540 & 640 & 690 & 530 \\
\hline 8 & 690 & 590 & 650 & 630 & 520 \\
\hline 9 & 670 & 470 & 680 & 695 & 480 \\
\hline
\end{tabular}

\section{RESULTADOS PROGRAMACIÓN DE CIRUGÍAS}

\section{CASO DE MÚLTIPLES OBJETIVOS}

Con los enfoques de solución modificados y considerando cada grupo por separado, se realiza la programación: día y pabellón de cirugías seleccionadas. Se desea una población de soluciones cercana a la frontera de Pareto. Para los grupos se obtienen las Figuras 1-6, que muestran a la población inicial y final con los cuatros tipos de operadores de selección.

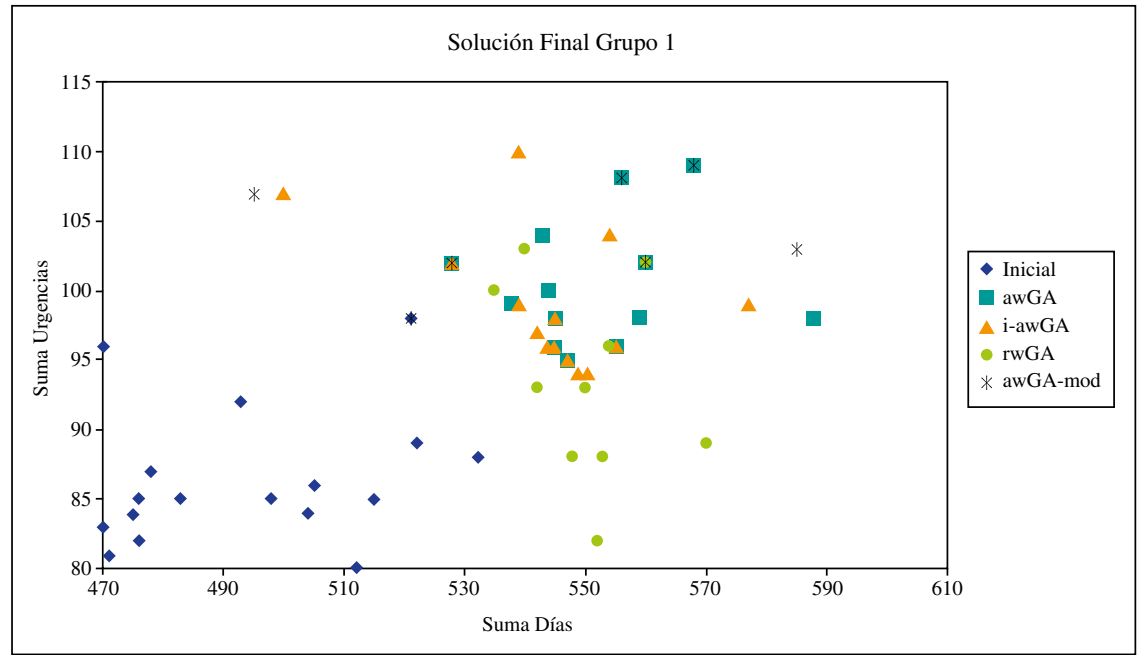

Figura 1. Conjunto Final Grupo 1.

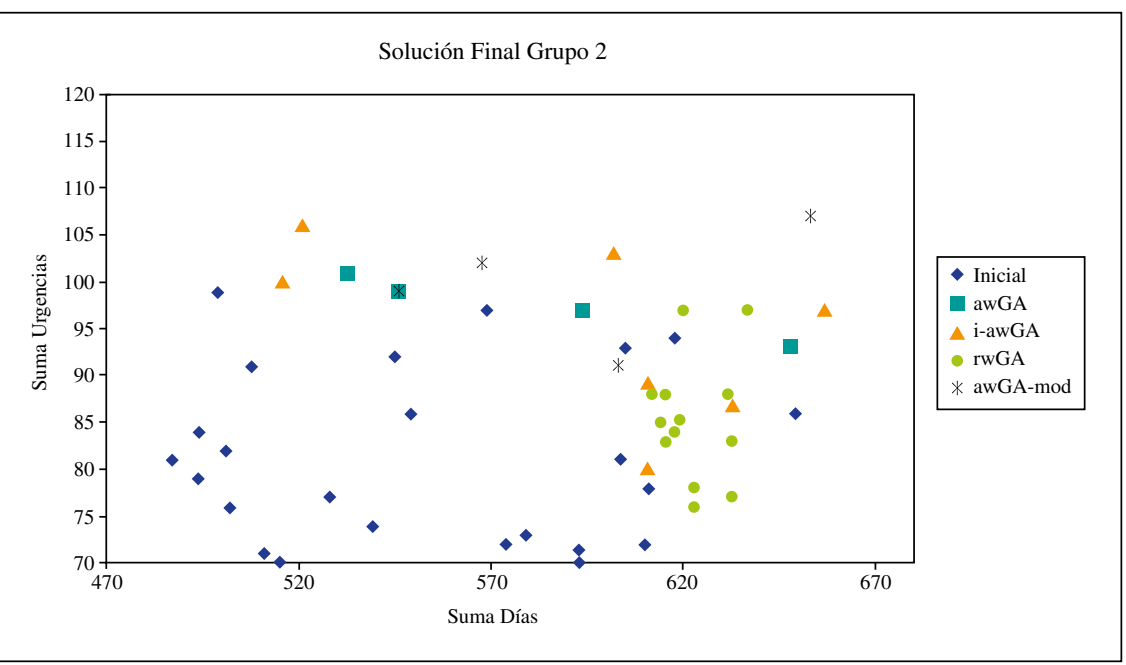

Figura 2. Conjunto Final Grupo 2. 


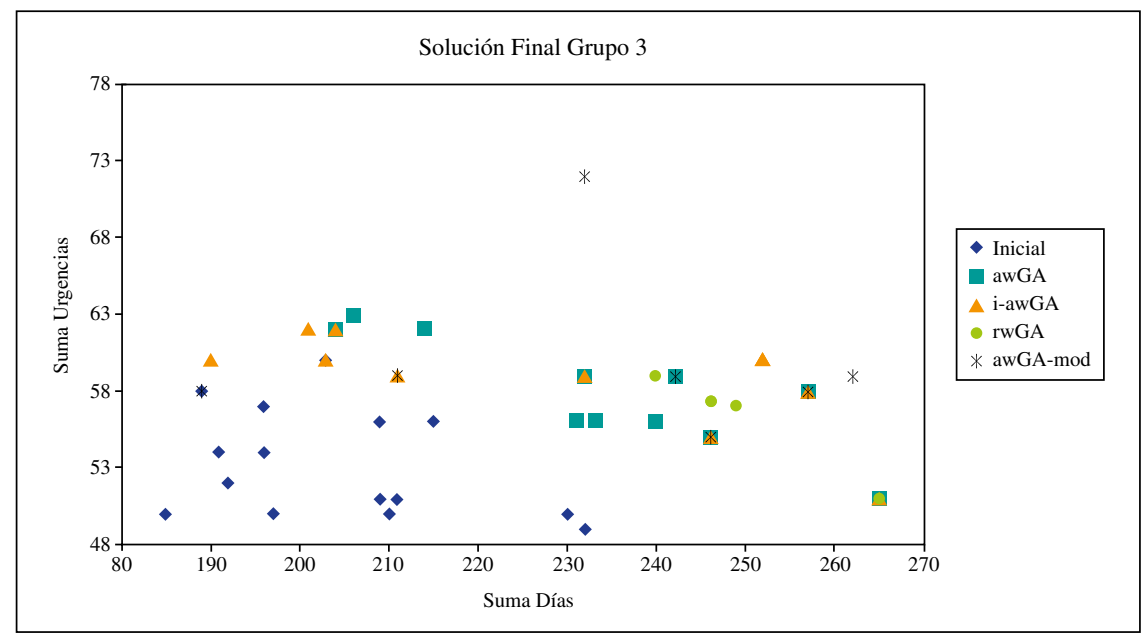

Figura 3. Conjunto Final Grupo 3.

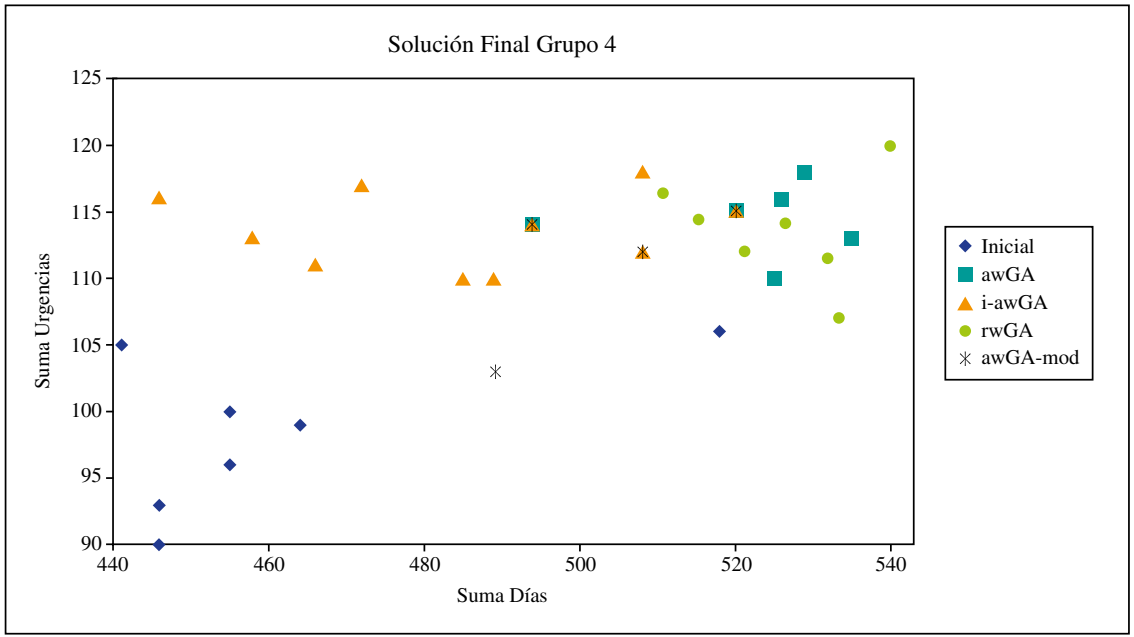

Figura 4. Conjunto Final Grupo 4.

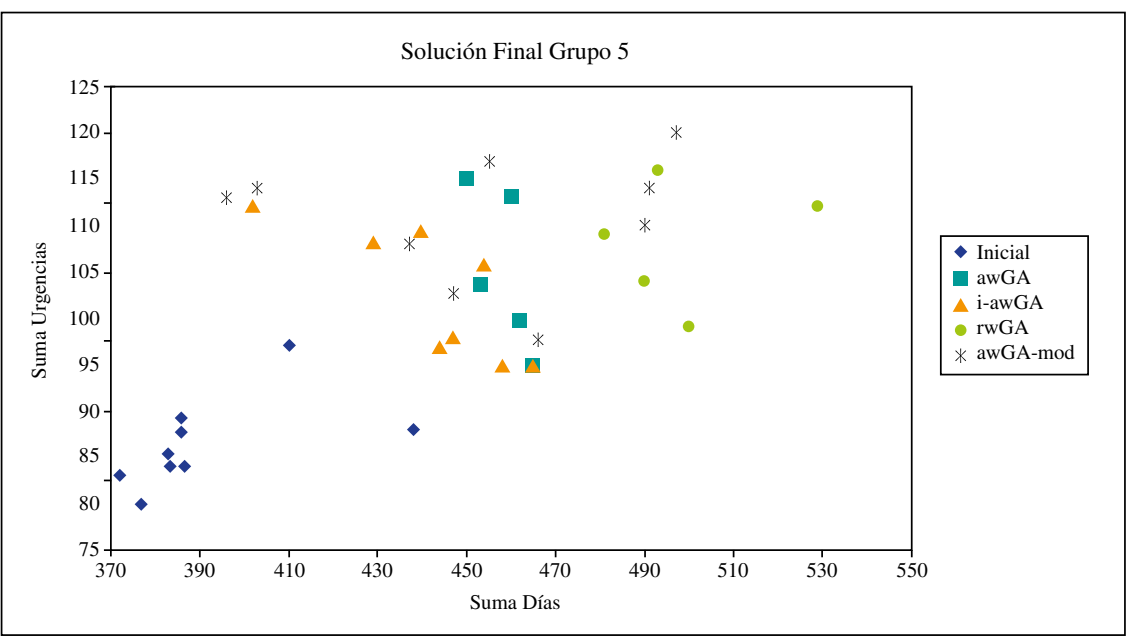

Figura 5. Conjunto Final Grupo 5. 


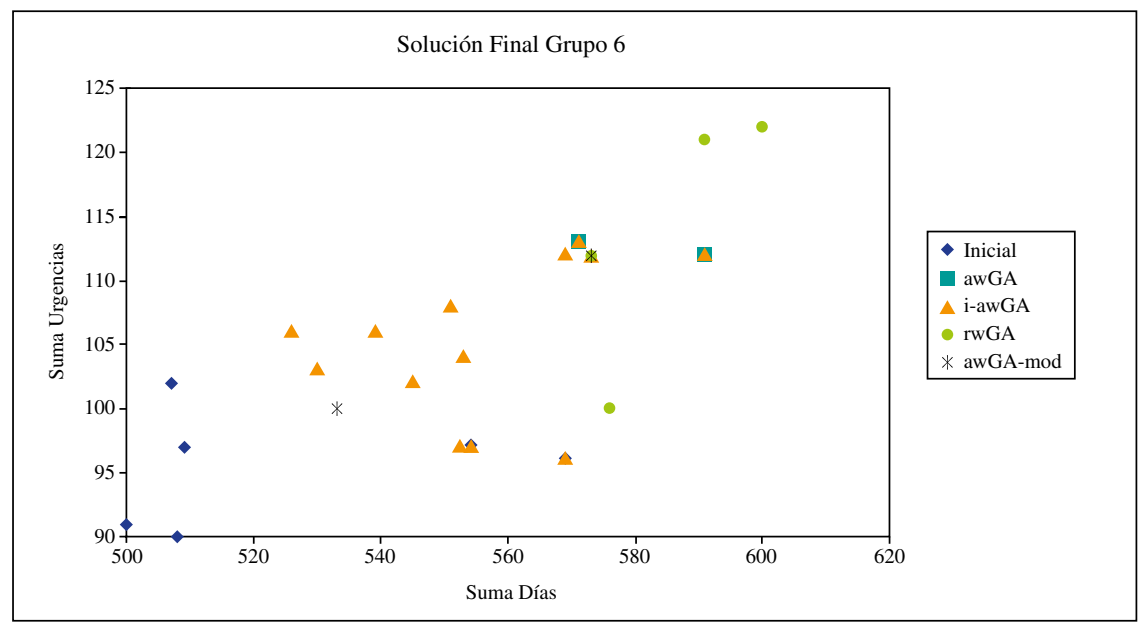

Figura 6. Conjunto Final Grupo 6.

En las figuras se observa la población inicial (rombos) y finales obtenidas por los métodos: $a w G A$, $i$-awGA, rwGA y awGA-mod. En los seis grupos se encuentran mejores soluciones, con valores más altos en ambos objetivos.

Los métodos de mejor desempeño son $a w G A$ y awGA-mod; en la mayoría de los grupos se obtienen mejores soluciones, exceptuando el grupo 6 donde $r w G A$ obtiene mejores soluciones en ambos objetivos.

\section{CASO DE UN OBJETIVO}

Considerando el enfoque metaheurístico de algoritmos genéticos con un solo objetivo se obtienen los resultados mostrados en la Tabla 4.

Tabla 4. Resultados considerando sólo un objetivo.

\begin{tabular}{|c|c|c|c|c|}
\cline { 2 - 5 } \multicolumn{1}{c|}{} & \multicolumn{2}{c|}{ Objetivo espera } & \multicolumn{2}{c|}{ Objetivo urgencia } \\
\hline Gr. & $\begin{array}{c}\text { Suma } \\
\text { espera }\end{array}$ & $\begin{array}{c}\text { Suma } \\
\text { urgencia }\end{array}$ & $\begin{array}{c}\text { Suma } \\
\text { espera }\end{array}$ & $\begin{array}{c}\text { Suma } \\
\text { urgencia }\end{array}$ \\
\hline 1 & 585 & 98 & 528 & 102 \\
\hline 2 & 624 & 94 & 565 & 105 \\
\hline 3 & 257 & 58 & 204 & 62 \\
\hline 4 & 571 & 118 & 508 & 123 \\
\hline 5 & 561 & 117 & 503 & 121 \\
\hline 6 & 600 & 103 & 573 & 112 \\
\hline
\end{tabular}

Con un objetivo se obtienen buenas soluciones, pero en algunos casos el objetivo que no es considerado alcanza un bajo valor, como por ejemplo el grupo 3, considerando solo el objetivo urgencia, la suma espera que está relacionada con el objetivo espera obtiene un valor de 204, que es muy bajo en comparación con los demás valores obtenidos, sin embargo, es una información adicional de utilidad para el tomador de decisiones al momento de determinar la programación de cirugías finales.

\section{ASIGNACIÓN PERSONAL ESPECIALIZADO}

A cada cirugía se asigna el personal requerido para su realización, el cual depende de cada grupo. Se utilizó un programa en lenguaje $\mathrm{C}++$, entregando como resultado el orden en el cual se efectuarían las cirugías y quién las realizaría, para los cinco días de programación.

Las cirugías son programadas en orden LPT (long process time), las de mayor tiempo de realización son programadas primero. La forma de asignar al personal es privilegiando el menor tiempo asignado acumulado, y así equilibrar la carga de trabajo del personal.

\section{CARGA LABORAL DEL PERSONAL}

La carga de trabajo es asignada de manera uniforme, con el fin de evitar que una persona tenga excesivas cirugías y evitar una baja utilización en comparación con el resto.

Para el análisis se usa una tabla con el porcentaje del total de la carga laboral asignado a una persona y en particular de grupos especializados. Valores mínimos y máximos se encuentran en la Tabla 5. 
Tabla 5. Resumen de carga laboral.

\begin{tabular}{|l|c|c|}
\hline Tipo de personal & Mínimo & Máximo \\
\hline Anestesistas & $9,94 \%$ & $10,10 \%$ \\
\hline Auxiliar anestesia & $4,69 \%$ & $4,86 \%$ \\
\hline Enfermeras & $4,93 \%$ & $5,09 \%$ \\
\hline Pabelloneras & $5,19 \%$ & $5,34 \%$ \\
\hline Arsenaleras & $5,19 \%$ & $5,34 \%$ \\
\hline Cirujano A & $12,10 \%$ & $13,12 \%$ \\
\hline Cirujano B & $9,57 \%$ & $10,45 \%$ \\
\hline Cirujano C & $10,47 \%$ & $12,07 \%$ \\
\hline Cirujano D & $10,83 \%$ & $11,53 \%$ \\
\hline Cirujano E & $12,06 \%$ & $13,28 \%$ \\
\hline Cirujano F & $9,63 \%$ & $10,39 \%$ \\
\hline Cirujano asistente A & $10,81 \%$ & $11,81 \%$ \\
\hline Cirujano asistente B & $8,01 \%$ & $8,83 \%$ \\
\hline Cirujano asistente C & $9,31 \%$ & $11,12 \%$ \\
\hline Cirujano asistente D & $8,00 \%$ & $8,74 \%$ \\
\hline Cirujano asistente E & $9,47 \%$ & $10,69 \%$ \\
\hline Cirujano asistente F & $7,83 \%$ & $8,83 \%$ \\
\hline
\end{tabular}

No existe diferencia entre valores mínimos y máximos de carga laboral, la carga es similar entre el personal.

\section{TIEMPOS DE EJECUCIÓN}

Con la configuración de pabellones y personal humano se comparan los tiempos de procesamiento utilizados por las cuatro estrategias de solución, ver Tabla 6.

Tabla 6. Tiempos de procesamiento.

\begin{tabular}{|c|c|c|c|c|c|}
\hline \multirow{2}{*}{ Gr. } & \multirow{2}{*}{$\mathbf{N}^{\circ}$ Cir. } & awGA & i-awGA & rwGA & $\begin{array}{c}\text { awGA- } \\
\text { mod }\end{array}$ \\
\cline { 3 - 6 } & & Tiempo[s] & Tiempo[s] & Tiempo[s] & Tiempo[s] \\
\hline 1 & 28 & 69,45 & 3520,31 & 263,93 & 550,56 \\
\hline 2 & 50 & 85,65 & 5853,28 & 469,68 & 510,28 \\
\hline 3 & 30 & 79,43 & 2981,47 & 321,75 & 485,14 \\
\hline 4 & 25 & 50,12 & 2576,48 & 311,55 & 341,59 \\
\hline 5 & 30 & 79,02 & 4957,13 & 584,64 & 344,98 \\
\hline 6 & 28 & 56,48 & 2325,98 & 330,56 & 490,65 \\
\hline Total (min.) & 191 & 7,0 & 370,2 & 38,0 & 45,4 \\
\hline
\end{tabular}

El método que presenta el menor tiempo de procesamiento es aw $G A$ con aproximadamente 7 minutos. El mayor tiempo de procesamiento con $370.2 \mathrm{~min}$ (6.17 horas) corresponde al método $i$ - $a w G A$.

\section{CONCLUSIONES}

En la mayoría de los centros de salud nacionales la programación de cirugías es manual, sólo se obtiene una única solución factible, y en algunos casos corresponde a una solución infactible, lo que trae como consecuencia la no realización de cirugías de listas programadas o un mal uso de los recursos.

Las metaheurísticas permiten mayor exploración del espacio de solución, encontrando buenas soluciones, $\mathrm{y}$ en el caso de los problemas multiobjetivo buenas aproximaciones de la frontera de Pareto.

Los tiempos de ejecución más bajos alcanzados son, para el método aw $G A$, cirugías, con un tiempo de 7 minutos, para las 191 .

El mejor método en calidad de soluciones y tiempo de ejecución es awGA, tiene mayor flexibilidad cuando existen contingencias, por ejemplo cirugías canceladas.

No es recomendable el método $i$-aw $G A$, por sus altos tiempos de procesamiento.

En el caso de la asignación de carga laboral, no se presenta sobrecarga de cirugías a una persona en particular (de cualquier especialidad) y se evita baja utilización del personal.

Se entregan buenas soluciones a bajo costo, debido a la utilización de Visual Express Edition (lenguaje $\mathrm{C}++)$, el cual es un software de uso libre.

El programa encontró buenas soluciones en los casos de dos y tres objetivos.

En futuros trabajos se recomienda realizar un estudio apropiado del tiempo que requiere cada cirugía y cómo una persona en particular puede influir en ese tiempo, se sugiere cuantificar la experiencia que pueda tener el cirujano y del tiempo que requiere un enfermo para responder a la anestesia.

\section{AGRADECIMIENTOS}

Este trabajo es parcialmente apoyado por el proyecto UDEC N ${ }^{\circ} 208.97011-1$ y Basal FB0816. 


\section{REFERENCIAS}

[1] R. Becerra. "Programación de salas quirúrgicas en un servicio de salud pública. Un enfoque multimochila para la solución". Informe de memoria de título para optar al título de Ingeniero Civil Industrial. Universidad de Concepción. Chile. 2006.

[2] B. Cardoen. "Operating room planning and scheduling: solving a surgical case sequencing problem". 4OR-Q J Oper Res Vol. 8, pp. 101-104. 2010. DOI 10.1007/ s10288-009-0106-z.

[3] A. Dietz. "Strategies for multiobjective genetic algorithm development: Application to optimal batch plant design in process systems engineering". Computers and Industrial Engineering. Vol. 54, pp. 539-569. 2008. ISSN: 0360-8352.

[4] P. Dinh-Nguyen and A. Klinker. "Surgical case scheduling as a generalized job shop scheduling problem". European Journal of Operational Research. Vol. 185, Issue 3, pp. 1011-1025. 2008. ISSN:0377-2217.

[5] C.M. Fonseca and P. J. Fleming. "Genetic algorithms for multiobjective optimization: Formulation, discussion and generalization". In Genetic Algorithms: Proceedings of the Fifth International Conference (S. Forrest, ed.). Morgan Kaufmann, pp. 416-423. San Mateo, CA, USA. 1993.

[6] M. Gen and R. Cheng. "Genetic Algorithms and Engineering Optimization". John Wiley \& Sons. New York. 2000. ISNB: 0-471-31531-1.

[7] F. Glover and M. Laguna. "Tabu Search”. In P.M. Pardalos and M.G.C. Resende (eds.), Handbook of Applied Optimization. Oxford University Press, pp. 194-208. New York, USA. 2002.

[8] J.H. Holland. "Adaptation in Natural and Artificial Systems". MIT Press. Cambridge, MA. 1992.
[9] H. Ishibuchi and T. Murata. "A multiobjective genetic local search algorithm and its application to flowshop scheduling". IEEE Transactions on Systems, Man and Cybernetics. Vol. 28, Issue 3, pp. 392-403. 1998.

[10] M. Lamiri, X. Xie, A. Dolgui and F. Grimaud. "A stochastic model for operating room planning with elective and emergency demand for surgery". European Journal of Operational Research. Vol. 185, Issue 3, pp. 1026-1037. 2008. ISSN:0377-2217.

[11] Z. Michalewicz and D.B. Fogel. "How to Solve It: Modern Heuristics". Springer,Verlag, Berling Heidelberg. Second Edition. New York. 2004. ISBN : 3-540-22494-7

[12] V. Pareto. "Manuale di Economica Polittica". Società Editrice Libraia. Milan, Italia. 1971.

[13] G. Pérez, M. Arenas Parra, A. Bilbao and M.V. Rodriguez. "Management of surgical waiting lists through a Possibilistic Linear Multiobjective Programming problem". Applied Mathematics and Computation. Vol. 167, Issue 1, pp. 477-495. 2005.

[14] B. Roland, C. Di Martinelly, F. Riane and Y. Pochet. "Scheduling an operating theatre under human resource constraints". Computers \& Industrial Engineering. Vol. 58, pp. 212-220. 2010. ISSN: 0360-8352.

[15] J.D. Schaffer. "Multiple objective optimization with vector evaluated genetic algorithms". Proceeding 1st International Conference on Gas, pp. 93-100. 1985.

[16] El-Ghazali Talbi. "Metaheuristics from design to implementation". John Wiley. New York. 2009. ISBN: 978-0-470-27858-1.

[17] F. Vidal. "Algoritmo de solución para problemas de programación de intervenciones quirúrgicas y asignación de cirujanos en el Hospital Regional de Concepción". Tesis para optar al grado de Magíster en Ingeniería Industrial. Universidad de Concepción. Chile. 2007. 